\title{
cmaJOPEN
}

\section{A comparison of faxed referrals and eConsult questions for rheumatology referrals: a descriptive study}

\author{
Erin Keely MD, Krista Rostom BSc(Eng) MD, Douglas Smith MD, Clare Liddy MD MSc
}

Abstract

Background: In Canada, wait times for access to specialized rheumatology services have increased, leading to new strategies to improve timely care; electronic consultations (eConsults) enable providers to ask specialists a clinical question using a secure platform, often reducing the need for a face-to-face visit. In this study, we sought to compare the types of referrals received through fax versus eConsult and to determine whether faxed referrals could be addressed using eConsult.

Methods: We conducted a descriptive study of consecutive faxed referrals sent to a tertiary care centre between Feb. 1 and Mar. 6 , 2017, and a convenience sample of eConsults directed to rheumatology between Feb. 1, 2015, and Sept. 30, 2016, through the Champlain BASE eConsult Service, an Ontario-based service. We reviewed all referrals and categorized them by clinical content and question type. A rheumatologist with experience completing eConsult referrals assessed faxed referrals for their suitability to be answered through eConsults. Descriptive statistics were generated.

Results: We analyzed 300 consecutive faxed referrals and 300 (of 470 ) eConsult referrals. Faxed questions more often pertained to rheumatoid arthritis (32/300 [10.7\%] v. 17/300 [5.7\%]), systemic lupus erythematosus (24/300 [8.0\%] v. 10/300 [3.3\%]), and polyarthritis $(30 / 300[10.0 \%]$ v. 18/300 [6.0\%]). eConsults more often addressed abnormal serology without joint symptoms (27/300 [9.0\%] v. $8 / 300$ [2.7\%]) and gout (15/300 [5.0\%] v. 4/300 [1.3\%]). Faxed referrals were more likely to have no specific question (116/300 $[38.7 \%])$, and eConsults were more likely to have more than 1 question posed $(99 / 300[33.0 \%])$ and a drug-related question $(67 / 300$ [22.3\%]). The rheumatologist identified potential benefit from eConsult in 216/300 (72.0\%) faxed referrals and 55/59 (93.2\%) declined faxed referrals.

Interpretation: Despite differences in diagnosis between eConsults and faxed referrals, most faxed referrals showed the potential to be addressed through eConsult. Using eConsult may allow primary care providers to obtain answers to questions without requesting a face-to-face specialist referral, or provide support for patients awaiting face-to-face consultation.

T he number of patients requiring care from a rheumatologist has increased in recent years, while the number of rheumatologists available to provide clinical care remains inadequate and static. ${ }^{1-3}$ In Canada, studies have found substantial variability in wait times for rheumatology. A study assessing time to first rheumatologist visit in new rheumatoid arthritis reported a mean wait time of 77 days, with a median wait time of 50 days. ${ }^{4}$ Another study reported a mean wait time of 155 (standard deviation 88) days for routine rheumatology referrals. ${ }^{5}$ To cope with these issues, some rheumatology practices have limited the number of referrals they accept and implemented strategies designed to improve triage, such as restrictions on accepted diagnoses, centralized intake and standardized referral forms. ${ }^{5-8}$ Unfortunately, these measures can lead to more declined referrals, which cause a greater burden for patients.

One solution in Eastern Ontario is the Champlain BASE (Building Access to Specialists Through eConsultation) eConsult Service, an asynchronous, secure Web-based appli- cation that allows primary care providers to submit patientspecific clinical questions to multiple specialty services, including rheumatology. ${ }^{9,10}$ User satisfaction is high, with primary care providers commonly citing the service's promptness, quality of replies and added education from specialist

Competing interests: Erin Keely and Clare Liddy are co-executive directors of the Ontario eConsult Centre of Excellence, funded by the Ontario Ministry of Health and Long-Term Care. They cofounded the Champlain BASE (Building Access to Specialists Through eConsultation) eConsult Service but do not retain any proprietary rights. Erin Keely answers eConsults through the service, less than 1 per month. After the study was completed, Krista Rostom began providing eConsult service through the Champlain BASE eConsult Service. Douglas Smith has completed eConsults through the Champlain BASE eConsult Service. No other competing interests were declared.

This article has been peer reviewed.

Correspondence to: Erin Keely, ekeely@toh.ca

CMAJ Open 2021. DOI:10.9778/cmajo.20200025 
responses as benefits. ${ }^{10,11}$ A study of 225 electronic consultations (eConsults) directed to rheumatology showed a median response time of 1.9 days, and found that $54 \%$ of cases resulted in a new course of action and $38 \%$ of cases resulted in the avoidance of an unnecessary referral. ${ }^{12}$

To understand eConsult's potential to improve access to rheumatology advice, in particular for providing advice for referrals that are declined, we need to identify whether the quality of information and types of clinical questions in faxed referrals (the usual means of requesting referrals in Ontario) lend themselves to being addressable through an eConsult. We aimed to determine whether there was an opportunity for eConsult to be used to provide advice for referrals that have been received through fax. We sought to compare the types of referrals received through fax versus a well-established eConsult service, and to determine which faxed referrals could likely be answered through an eConsult without a face-to-face visit, and in which cases an eConsult could provide advice through preconsultation exchange while the patient is waiting for the face-to-face visit.

\section{Methods}

\section{Study design}

We conducted a descriptive study of eConsults and faxed referrals sent to rheumatology.

\section{Setting and participants}

The study contained 2 distinct samples — faxed referrals and eConsults - both of which occurred in the Champlain health region located in Eastern Ontario, Canada, with a population of about 1.2 million people.

Faxed referrals received between Feb. 1 and Mar. 6, 2017, were collected from the Division of Rheumatology at The Ottawa Hospital, a tertiary care centre within the Champlain region, which at the time of this study had 6 full-time academic rheumatologists. Requests for referral are usually faxed to a central number and reviewed on a rotating schedule by a rheumatologist. The faxed referral is either accepted or declined on the basis of the rheumatologist's review. This triage decision is noted on a cover sheet.

There are currently 2 models for incorporating eConsults into the referral pathway. In the BASE model, the primary care provider chooses to either submit a request for an eConsult or fax a request to a central triage system. If, when the eConsult has been completed, the primary care provider chooses to refer, they can do so using their usual workflow. However, the appointment may not be with the person who completed the eConsult. In services with an integrated eReferral system, all patient referrals are submitted electronically, and the specialist has the option of responding electronically (i.e., through eConsult) or booking the patient for an in-person appointment.

In this study, eConsults came from the Champlain BASE eConsult Service, which was available to all primary care providers across the Champlain region. Specialists are expected to respond within 1 week, providing a recommendation, sug- gesting a face-to-face referral or requesting further information. Specialists are paid based on a prorated hourly rate. They self-report the billing time per case. When the referring provider has received the advice, they must complete a mandatory 4-question survey (Appendix 1, available at www. cmajopen.ca/content/9/1/E38/suppl/DC1). As of September 2020, this eConsult service had completed more than 64000 cases, enrolled more than 1700 providers and provided access to 135 specialty groups. ${ }^{13}$

\section{Data collection}

Our sample size was 300 faxed referrals and 300 eConsults. Consecutive faxed referrals were collected between Feb. 1 and Mar. 6, 2017, to achieve a convenience sample of 300 referrals. The triage decision made independently of this study was captured. For eConsult, 470 referrals were submitted to rheumatology between Feb. 1, 2015, and Sept. 30, 2016. From the 470, the first 300 referrals in the data set were selected for analysis. As the referrals in the data set were not ordered by date, the 300 eConsults selected for this study were not consecutive.

For both faxed referrals and eConsults, the patient's sex and age, and referring provider type (e.g., nurse practitioner, family physician, specialist) were collected. In addition to a complete log of the exchange between primary care provider and specialist, eConsults include utilization data collected automatically by the service (e.g., the time it took for the primary care provider to get a response from the specialist and time the specialist self-reported as their billing time to complete the case) and primary care provider responses to a mandatory closeout survey assessing the eConsult's impact on course of action and primary care provider decision to refer.

All faxed referrals and eConsults were reviewed and categorized retrospectively by clinical content and type of question by a single reviewer (K.R.) using a predefined list of 31 clinical diagnoses and types of clinical question content. ${ }^{14}$ The broad categories for clinical question content included diagnosis, drug related, procedure, management, epidemiology, and nonclinical or administrative questions. If the consult asked 2 unique clinical content questions, it was classified as "more than 1 specific question." If the consult did not ask a specific question that could be classified based on the clinical content categories, it was classified as "no specific question."

Each faxed referral was assessed for suitability for an eConsult by a rheumatologist (D.S.) who regularly completes eConsults. The rheumatologist chose 1 of 4 options: 1) I could answer this consultation with an eConsult, thus likely avoiding a face-to-face consultation, 2) I could possibly answer this consultation with an eConsult, but information is missing, 3) I could provide some advice regarding this consultation via eConsult, but the patient would still likely require a face-to-face consultation, 4) I would be unable to provide advice via eConsult. The rheumatologist was blinded to the triage outcome of the faxed referral. Within each of the above 4 options, the rheumatologist could then choose more specific reasons to qualify his choice (e.g., "requires physical exam to address the question" or "requires rheumatologist to follow patient due to complexity/standard of care"). 


\section{Statistical analysis}

Descriptive statistics were generated for patient and provider characteristics and other variables for eConsults and faxed referrals, using Excel.

\section{Ethics approval}

Ethics review exemption was granted under the Quality Assurance project protocol from the Ottawa Hospital Research Ethics Board.

\section{Results}

\section{Description of faxed referrals}

Of the 300 consecutive faxed referrals collected during the study period, $201(67.0 \%)$ were sent from a primary care provider and 97 (32.3\%) from a specialist. Referrals were sent from 150 different primary care providers, with 33 primary care providers sending 2 or more referrals. Of the primary care providers who referred patients by faxed referral, $58(38.7 \%)$ had an eConsult account at the time they completed the faxed referral. Table 1 summarizes the referral source and patient characteristics.

Complete data specifically on the triaging of the faxed referrals were available for 290 referrals. Faxed referrals were triaged within a median of 5 (interquartile range 2-7) days from when the consult was received. However, the wait time for patients to receive an appointment depended on how the referral was triaged, with $6(2.1 \%)$ of the 290 referrals with complete data triaged to urgent $(<2 \mathrm{wk}), 50(17.2 \%)$ to semiurgent (2-8 wk), $122(42.1 \%)$ to routine $(3-6 \mathrm{mo})$ and 53 $(18.3 \%)$ to routine $(\geq 6 \mathrm{mo})$.

Overall, 59 (19.7\%) of faxed referrals were declined, with $52(88.1 \%)$ of these originating from a primary care provider. Referrals were declined for various reasons: 21 (35.6\%) were deemed to not have enough information, 25 (42.4\%) were for a diagnosis not accepted by the clinic (e.g., osteoarthritis, fibromyalgia and back pain), and 13 (22.0\%) at the discretion of the rheumatologist, without a reason provided.

\section{Description of eConsults}

Of the 15757 eConsults completed from February 2015 to September 2016, 470 (3.0\%) were directed to rheumatology, and 300 of these eConsults were analyzed. Two rheumatologists answered most referrals (115 [38.3\%] and 118 [39.3\%]), a third rheumatologist answered 66 (22.0\%) and a fourth rheumatologist answered 1 referral. For 60 referrals $(20.0 \%)$, the rheumatologist requested further information from the primary care provider before answering. Self-reported time to complete the eConsult was less than 10 minutes in 147 (49.0\%), 10-15 minutes in 120 (40.0\%), 15-20 minutes in 27 $(9.0 \%)$ and more than 20 minutes in $6(2.0 \%)$ referrals.

The median time for the primary care provider to receive a response from the rheumatologist through the eConsult service was 1 day (range $10 \mathrm{~min}$ to $9.4 \mathrm{~d}$ ). eConsult advice changed the course of action for 148 (49.3\%) patients and allowed the primary care provider to confirm a course of action they had in mind in 138 (46.0\%) cases. In $116(38.7 \%)$ cases, a referral was originally considered but avoided based on the specialist's advice, and in 94 (31.3\%) cases the referral originally considered was still completed. A total of $9(3.0 \%)$ primary care providers felt there was no particular benefit to eConsult in their case.

\section{Comparison of eConsult and faxed referrals}

Both the faxed and eConsult questions included a diverse set of diagnoses and symptoms (Table 2). Osteoporosis was a common cause for consultation in both referral methods, accounting for 28 (9.3\%) faxed referrals and $51(17.0 \%)$ eConsults. Faxed referrals more often pertained to rheumatoid arthritis (32 [10.7\%] v. 17 [5.7\%]), systemic lupus erythematous $(24[8.0 \%]$ v. $10[3.3 \%])$ and polyarthritis $(30$ $[10.0 \%]$ v. $18[6.0 \%])$. eConsult cases more often involved abnormal serology without joint symptoms (27 [9.0\%] v. 8 [2.7\%]) and gout (15 [5.0\%] v. 4 [1.3\%]).

Question type was similar between faxed referrals and eConsults, with a few notable differences. Faxed referrals were more likely to have no specific question (116 [38.7\%]), and

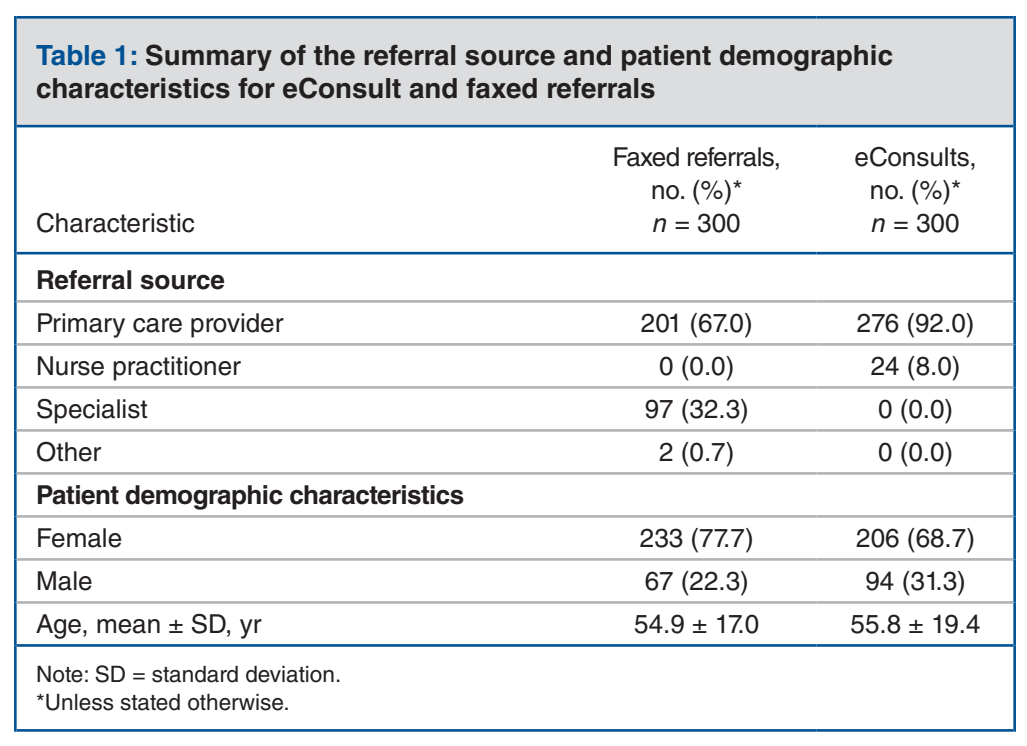




\begin{tabular}{|c|c|c|}
\hline \multicolumn{3}{|c|}{$\begin{array}{l}\text { Table 2: Clinical content and question type for faxed referrals and } \\
\text { eConsults }\end{array}$} \\
\hline Variable & $\begin{array}{c}\text { Faxed referrals, } \\
\text { no. }(\%) \\
n=300\end{array}$ & $\begin{array}{c}\text { eConsults, } \\
\text { no. (\%) } \\
n=300\end{array}$ \\
\hline \multicolumn{3}{|l|}{ Clinical content } \\
\hline Abnormal serology without joint symptoms & $8(2.7)$ & $27(9.0)$ \\
\hline Bursitis & $1(0.3)$ & $0(0.0)$ \\
\hline Connective tissue disease & $9(3.0)$ & $3(1.0)$ \\
\hline Crystal arthropathy - pseudogout & $2(0.7)$ & $1(0.3)$ \\
\hline Crystal arthropathy — gout & $4(1.3)$ & $15(5.0)$ \\
\hline Elevated creatine kinase & $1(0.3)$ & $7(2.3)$ \\
\hline Fibromyalgia & $8(2.7)$ & $3(1.0)$ \\
\hline Inflammatory monoarthritis & $5(1.7)$ & $12(4.0)$ \\
\hline Inflammatory polyarthritis & $30(10.0)$ & $18(6.0)$ \\
\hline Osteoarthritis & $13(4.3)$ & $9(3.0)$ \\
\hline Osteoporosis & $28(9.3)$ & $51(17.0)$ \\
\hline Paget disease of bone & $1(0.3)$ & $3(1.0)$ \\
\hline Pain - back & $4(1.3)$ & $11(3.7)$ \\
\hline Pain - multiple joints & $28(9.3)$ & $21(7.0)$ \\
\hline Pain - not otherwise specified & $3(1.0)$ & $2(0.7)$ \\
\hline Pain - single joint & $1(0.3)$ & $4(1.3)$ \\
\hline Polymyalgia rheumatica & $12(4.0)$ & $19(6.3)$ \\
\hline Raynaud disease & $4(1.3)$ & $9(3.0)$ \\
\hline Rheumatoid arthritis & $32(10.7)$ & $17(5.7)$ \\
\hline Scleroderma & $5(1.7)$ & $0(0.0)$ \\
\hline Sjögren syndrome & $7(2.3)$ & $4(1.3)$ \\
\hline Systemic lupus erythematous & $24(8.0)$ & $10(3.3)$ \\
\hline Vasculitis & $12(4.0)$ & $7(2.3)$ \\
\hline Other & $38(12.7)$ & $40(13.3)$ \\
\hline No specific diagnosis given & $9(3.0)$ & $0(0.0)$ \\
\hline \multicolumn{3}{|l|}{ Question type } \\
\hline Diagnosis & $75(25.0)$ & $72(24.0)$ \\
\hline Drug related & $25(8.3)$ & $67(22.3)$ \\
\hline Procedure & $6(2.0)$ & $3(1.0)$ \\
\hline Management & $61(20.3)$ & $58(19.3)$ \\
\hline Epidemiology & $0(0.0)$ & $1(0.3)$ \\
\hline Nonclinical or administrative & $2(0.7)$ & $0(0.0)$ \\
\hline More than 1 specific question & $15(5.0)$ & $99(33.0)$ \\
\hline No specific question & $116(38.7)$ & $0(0.0)$ \\
\hline
\end{tabular}

eConsults were more likely to have more than 1 question posed by the primary care provider $(99$ [33.0\%]) and a drugrelated question (67 [22.3\%]) (Table 2).

\section{Suitability for eConsult}

Among the referrals submitted by fax, the participating rheumatologist identified $59(19.7 \%)$ that could likely be answered through eConsult and $62(20.7 \%)$ that could possibly be answered through eConsult, as well as 96 (32.0\%) for which some advice could be provided through eConsult though the patient would likely still need a face-to-face consultation.

Among the 59 declined faxed referrals, the rheumatologist determined that eConsult would be beneficial in 55 cases (93.2\%), including 32 cases (54.2\%) in which the rheumatologist could likely provide a response through eConsult, 18 cases $(30.5 \%)$ in which he could possibly provide a response through eConsult, and 5 cases $(8.5 \%)$ in which he could provide advice on treatment though a face-to-face consultation 
Table 3: Suitability of the faxed referrals for eConsult, as assessed by a rheumatologist*

\begin{tabular}{|c|c|}
\hline Response & $\begin{array}{l}\text { No. }(\%) \\
n=300\end{array}$ \\
\hline $\begin{array}{l}\text { I could likely answer this consultation with an eConsult, thus likely avoiding a } \\
\text { face-to-face consultation }\end{array}$ & $59(19.7)$ \\
\hline $\begin{array}{l}\text { I could possibly answer this consultation with an eConsult, but information is } \\
\text { missing: }\end{array}$ & $62(20.7)$ \\
\hline $\begin{array}{l}\text { Requires further history to address this question, which could be obtained } \\
\text { with exchange via eConsult }\end{array}$ & $54 / 62(87.1)$ \\
\hline $\begin{array}{l}\text { Requires further investigations to address this question, which could be } \\
\text { obtained with exchange via eConsult }\end{array}$ & $50 / 62(80.6)$ \\
\hline $\begin{array}{l}\text { I could provide some advice regarding this consultation via eConsult, but the } \\
\text { patient still likely requires a face-to-face consultation because }\end{array}$ & $96(32.0)$ \\
\hline Requires physical exam to address the question & $62 / 96(64.6)$ \\
\hline $\begin{array}{l}\text { Requires further investigations (blood work, imaging, etc.) to address the } \\
\text { question, which is not amenable to eConsult exchange }\end{array}$ & $23 / 96(24.0)$ \\
\hline $\begin{array}{l}\text { Requires rheumatologist to follow patient owing to complexity/standard of } \\
\text { care for given diagnosis/chronic drug therapy that requires rheumatology } \\
\text { expertise }\end{array}$ & $35 / 96(36.5)$ \\
\hline Referred by another specialist & 18/96 (18.8) \\
\hline Likely requires a procedure by a rheumatologist & 2/96 (2.1) \\
\hline Other & $0(0)$ \\
\hline I am unable to provide advice via eConsult, because & $83(27.7)$ \\
\hline No clear question to be answered & 2/83 (2.4) \\
\hline Requires physical exam to address the question & 16/83 (19.3) \\
\hline $\begin{array}{l}\text { Requires further investigations (blood work, imaging, etc.) to address the } \\
\text { question, which is not amenable to eConsult exchange }\end{array}$ & $6 / 83(7.2)$ \\
\hline $\begin{array}{l}\text { Requires rheumatologist to follow patient owing to complexity/standard of } \\
\text { care for given diagnosis/chronic drug therapy that requires rheumatology } \\
\text { expertise }\end{array}$ & $65 / 83(78.3)$ \\
\hline Referred by another specialist & $47 / 83(56.6)$ \\
\hline Likely requires a procedure by a rheumatologist & $1 / 83(1.2)$ \\
\hline Other & $1 / 83(1.2)$ \\
\hline
\end{tabular}

would likely still be needed (Table 3 ). In 4 cases $(6.8 \%)$, the rheumatologist felt that he would be unable to provide advice by eConsult.

\section{Interpretation}

In our study, the majority of faxed referrals showed the potential to be at least initially addressed through eConsult. Whereas $19.7 \%$ of faxed referrals were declined, the rheumatologist identified $93.2 \%$ of this subset as potentially answerable through eConsult. This finding suggests that eConsult, if effectively integrated into clinician workflows, could serve as a powerful tool to mitigate the delays and frustrations associated with declined referrals, as rheumatologists unable to accept the referral would have the option of providing advice and support to the primary care provider directly.

In the literature, both the BASE model and the eReferral system have been found to improve communication between primary care providers and specialists, resulting in unnecessary referrals being avoided. For instance, a study of rheumatology cases submitted to the eReferral service at the San Francisco General Hospital found that most referrals (74\% in the last half of the study) involved a preconsultation exchange between the specialist and primary care provider, which usually included a request for further testing or clarification of clinical information. ${ }^{15}$ Of these, only $63 \%$ ultimately required a face-to-face visit. Similarly, our previous study showed that $38 \%$ of rheumatology eConsults resulted in avoidance of a face-to-face referral. ${ }^{12}$

The results of our current study show that if we were to apply eConsult to faxed consults to facilitate communication between rheumatologists and primary care providers, we could possibly help to reduce unacceptably long wait times, remove the frustration of not receiving any advice or support on declined referrals, and make face-to-face consults more effective. 
When seeking to implement solutions to long wait times, it is important to consider their impact on clinical workload and satisfaction for primary care providers and specialists alike. In both the BASE and San Francisco services, ${ }^{12,15}$ a small number of rheumatologists provided the bulk of eConsult responses. The time taken to answer the question is almost always less than 15 minutes. ${ }^{12}$ Rather than limiting access through declining referrals, eConsult services allow specialists to provide guidance and education to referring providers. A recent Canadian study by Widdifield and colleagues reported that, among patients with new rheumatoid arthritis, the median time from initial symptom onset documented by primary care provider to rheumatology consultation was 327 days, and the median time from primary care provider referral to rheumatology consult was 66 days more than twice the current wait time benchmark from the Canadian Rheumatology Association. ${ }^{16,17}$ eConsult has the potential to decrease wait times for those who need a face-to-face referral while increasing primary care provider knowledge and communication between primary care providers and specialists, which has been shown to improve rheumatology referrals. ${ }^{18}$

\section{Limitations}

Our study has limitations. Our study was completed in 1 region, at 1 academic centre, and with 1 rheumatologist who reviewed faxed consults for suitability for eConsult. Additionally, we used a convenience sample to collect eConsult referrals, as the data set was not ordered by date. Therefore, our results may not be generalizable to other settings. A single rheumatologist, experienced in both in-person referrals and eConsults, determined whether a faxed referral would be suitable as an eConsult. We cannot say whether other rheumatologists would agree with his perspective. As well, $32.3 \%$ of faxed referrals were sent from specialists, who generally do not send referrals via eConsult and may be unwilling to action advice they would receive through an eConsult if it falls outside of their scope of practice. There was a difference in the time frame of the eConsults versus faxed referrals owing to logistics. This is unlikely to have altered the types of questions received.

\section{Conclusion}

In our study, most faxed referrals showed the potential to be at least initially addressed through eConsult, which is especially important for those that would otherwise be rejected. Our study identifies an opportunity to provide support to primary care providers through eConsults for patients who would meet the criteria to be seen in rheumatology clinics, as well as for cases in which access is denied. Primary care providers and specialists should consider using eConsult services to improve access to timely specialist advice.

\section{References}

1. Hanly JG; Canadian Council of Academic Rheumatologists. Manpower in Canadian academic rheumatology units: current status and future trends. Canadian Council of Academic Rheumatologists. 7 Rheumatol 2001;28:1944-51.

2. Hanly JG. Physician resources and postgraduate training in Canadian academic rheumatology centers: a 5-year prospective study. 7 Rheumatol 2004:31:1200-5.

3. Widdifield J, Paterson JM, Bernatsky S, et al. The rising burden of rheumatoid arthritis surpasses rheumatology supply in Ontario. Can $\mathcal{F}$ Public Health 2013;104:e450-5.
4. Widdifield J, Paterson JM, Bernatsky S, et al. Access to rheumatologists among patients with newly diagnosed rheumatoid arthritis in a Canadian universal public healthcare system. BM7 Open 2014;4:e003888.

5. Hazlewood GS, Barr SG, Lopatina E, et al. Improving appropriate access to care with central referral and triage in rheumatology. Artbritis Care Res (Hoboken) 2016;68:1547-53.

6. Gran JT, Nordvåg BY. Referrals from general practice to an outpatient rheumatology clinic: disease spectrum and analysis of referral letters. Clin Rheumatol 2000;19:450-4.

7. Thompson AE, Haig SL, LeRiche NG, et al. Comprehensive arthritis referral study - phase 2: analysis of the comprehensive arthritis referral tool. 7 Rheumatol 2014;41:1980-9.

8. Farrer C, Abraham L, Jerome D, et al. Triage of rheumatology referrals facilitates wait time benchmarks. 7 Rheumatol 2016;43:2064-7.

9. Keely E, Liddy C, Afkham A. Utilization, benefits, and impact of an e-consultation service across diverse specialties and primary care providers. Telemed F E Health 2013;19:733-8.

10. Liddy C, Rowan MS, Afkham A, et al. Building access to specialist care through e-consultation. Open Med 2013;7:e1-8.

11. Liddy C, Afkham A, Drosinis P, et al. Impact and satisfaction with a new eConsult service: a mixed methods study of primary care providers. $7 \mathrm{Am}$ Board Fam Med 2015;28:394-403.

12. Rostom K, Smith CD, Liddy C, et al. Improving access to rheumatologists: use and benefits of an electronic consultation service. 7 Rheumatol 2018;45:137-40.

13. eConsult by the numbers. Champlain BASE eConsult. Available: https://221cf17f-efad-46eb-9bec-9296ed538058.filesusr.com/ugd/ac5147_1b 98d08a8ef54af591840130b6f46693.pdf (accessed 2020 Aug. 24).

14. Ely JW, Osheroff JA, Gorman PN, et al. A taxonomy of generic clinical questions: classification study. BM7 2000;321:429-32.

15. Scheibe MM, Imboden JB, Schmajuk G, et al. Efficiency gains for rheumatology consultation using a novel electronic referral system in a safety net health setting. Artbritis Care Res (Hoboken) 2015;67:1158-63.

16. Widdifield J, Tu K, Carter Thorne J, et al. Patterns of care among patients referred to rheumatologists in Ontario, Canada. Artbritis Care Res (Hoboken) 2017;69:104-14

17. Canadian Rheumatology Association. Wait-time benchmarks for rheumatology. Available: www.waittimealliance.ca/wp-content/uploads/2014/05/Wait-Time -Benchmarks-for-Rheumatology-FINAL.pdf (accessed 2020 Dec. 16).

18. Schulpen GJC, Vierhout WPM, Van der Heijde DM, et al. Joint consultation of general practitioner and rheumatologist: Does it matter? Ann Rheum Dis 2003;62:159-61.

Affiliations: Department of Medicine (Keely, Smith), University of Ottawa; Department of Medicine (Keely, Smith) and Ontario eConsult Centre of Excellence (Keely, Liddy), The Ottawa Hospital; Department of Medicine (Rostom), Queensway Carleton Hospital; Department of Family Medicine (Liddy), C.T. Lamont Primary Health Care Research Centre, University of Ottawa; Bruyère Research Institute (Liddy), Ottawa, Ont.

Contributors: Erin Keely and Clare Liddy conceived the study and design. Krista Rostom and Douglas Smith acquired the data. Krista Rostom, Douglas Smith, Erin Keely and Clare Liddy analyzed and interpreted the data. Krista Rostom and Erin Keely drafted the manuscript. All authors contributed to the drafting and editing of the manuscript, and gave final approval of the version to be published. All authors agree to be accountable for all aspects of the work.

Funding: Funding for this study was provided by the University of Ottawa Department of Medicine and the Royal College of Physicians and Surgeons of Canada.

Content licence: This is an Open Access article distributed in accordance with the terms of the Creative Commons Attribution (CC BY-NC-ND 4.0) licence, which permits use, distribution and reproduction in any medium, provided that the original publication is properly cited, the use is noncommercial (i.e., research or educational use), and no modifications or adaptations are made. See: https://creativecommons.org/licenses/by-nc-nd/4.0/

Data sharing: The data set contains personal health and identifying information and cannot be shared for privacy reasons. An anonymized version of the data used in this study can, where it is possible to ensure privacy, be made available on reasonable request.

Acknowledgements: The authors thank the providers who use the eConsult service, and Justin Joschko and Jessika Hammond for their assistance in preparing the manuscript for publication.

Supplemental information: For reviewer comments and the original submission of this manuscript, please see www.cmajopen.ca/content/9/1/ E38/suppl/DC1. 